RESEARCH PAPER RP1614

Part of Journal of Research of the National Bureau of Standards, Volume 33,

November 1944

\title{
ATTACK OF REFRACTORY PLATINIFEROUS MATERIALS BY ACID MIXTURES AT ELEVATED TEMPERATURES
}

\author{
By Edward Wichers, William G. Schlecht, and Charles L. Gordon
}

\section{ABSTRACT}

Iridium and other refractory metals and alloys of the platinum group can be easily prepared for analysis, or for small-scale refining, by solution with hydrochloric acid and suitable oxidants in sealed tubes at temperatures up to $300^{\circ} \mathrm{C}$. The effects on the rate of solution of iridium caused by variations in temperature, in concentrations of hydrochloric acid and of the oxidant, and in the nature of the oxidant, are reported. The rate increases rapidly with increase in temperature. It also increases with increasing concentration of hydrochloric acid, up to nearly anhydrous hydrogen chloride. With variation in the concentration of chlorine or equivalent oxidants, the rate passes through a maximum at a relatively low concentration.

A number of other factors of less importance are also discussed. Suitable working conditions for dissolving various alloys and minerals are suggested.

\section{CONTENTS}

II. Apparatus, reagents, and experimental procedure $\ldots \ldots \ldots \ldots . . . . . .364$

III. Experimental results

1. Effect of variation in temperature $\ldots \ldots \ldots 8$

2. Effect of variation in the concentration of hydrogen chloride $\ldots 368$

3. Variation in the concentration of chlorine _...

4. Miscellaneous observations _._. _ _ _ _ _ 376

5. Preparation of samples for analysis $\ldots \ldots \ldots \ldots \ldots \ldots$

IV. Best operating conditions _ _

1. Quantity and strength of hydrochloric acid

2. Nature and quantity of oxidizing agent $\ldots \ldots \ldots$

3. Temperature 380

4. Dimensions of reaction tube

5. Protection and handling of the sealed tubes

\section{INTRODUCTION}

Of the six platinum metals only platinum and palladium can be dissolved in aqua regia at temperatures that can be reached in open vessels. The other four and their alloys, such as osmiridium, are nearly unattacked by aqua regia at temperatures up to the boiling point of the acid mixture. In preparing platinum-group minerals for analysis, the customary procedure is to treat them with aqua regia until no more dissolves, and then to worry the undissolved residue more or less completely into soluble form by repeated treatments with alkaline oxidizing fluxes. This process is not only laborious, but may result in losses 


\section{Journal of Research of the National Bureau of Standards}

of material and in contamination from various sources. Further, the solution eventually obtained contains an undesirable amount of alkali salts. Besides the refractory platiniferous minerals, some manufactured platinum alloys also contain enough rhodium or iridium to make them very difficultly soluble in aqua regia. They are even more difficult to treat by fusion with alkalies.

The stability of the chloro compounds of the platinum metals suggests that the difficulty encountered in dissolving some of the metals in hydrochloric acid plus a suitable oxidizing agent, is not caused by the nature of the reaction but by the rate at which the reaction proceeds at the temperatures ordinarily used. This idea is supported by the fact that a given mass of one of these metals is more rapidly dissolved if the sample is very finely divided, or if the sample of refractory metal is alloyed with a sufficiently large proportion of platinum. In both cases the rate of attack per unit area of exposed surface remains low, but the amount of metal going into solution is increased because the effective surface area is increased. It therefore seemed worth while to find out whether the rate of attack could be sufficiently increased for practical purposes at temperatures attainable in glass apparatus.

\section{APPARATUS, REAGENTS, AND EXPERIMENTAL PROCEDURE}

The pressure developed by concentrated hydrochloric acid or its mixtures with nitric acid or other oxidizing agents increases very rapidly above $100^{\circ} \mathrm{C}$. In the observations to be reported, pressures up to $4,000 \mathrm{lb}$./in. ${ }^{2}$ were often encountered. Well-annealed sealed glass tubes normally will withstand such pressures if the internal diameter is not greater than twice the wall thickness. Pyrex glass tubes of 4-mm diameter and 2-mm wall thickness were used for nearly all the experimental work on rates of solution, with no protection except inclosure in a loosely capped piece of pipe, which served to keep glass from scattering about the oven when an occasional failure occurred. These tubes usually were about $20 \mathrm{~cm}$ long. For a few similar experiments, Pyrex tubes of 8-mm bore and 2-mm wall thickness were used. For the solution of minerals and other materials to be prepared for, analysis, tubes of $15-\mathrm{mm}$ bore, with about $2.5 \mathrm{~mm}$ wall thickness, were used. The latter tubes, also about $20 \mathrm{~cm}$ long, were provided with stems of 4- by 8-mm tubing through which the reaction mixture was introduced (fig. 1). The sample was introduced through the neck unless the size of the pieces, such as nuggets of mineral, made it necessary to place them in the larger tube before the stem was sealed on. The reason for using tubes provided with stems of smaller bore has been discussed in a previous paper. ${ }^{1}$ In brief, experience showed that the larger tubes could

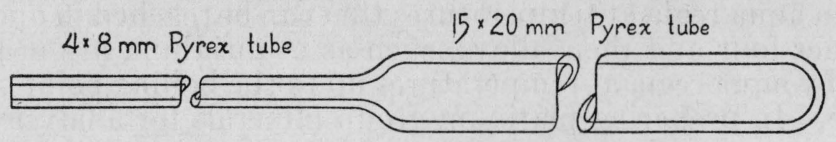

Figdre 1.-Sealed tube for solutions.

The volume of the main tube is approximately $1.77 \mathrm{ml} / \mathrm{cm}$. The tube is made twice the length that will accommodate the desired amount of reacting mixture.

1 C. L. Gordon, J. Research NBS 30, 107-111 (1943) RP1521. 
not be sealed, after introducing the sample and acid mixture, without leaving the seals so strained as to cause frequent failures at relatively low pressures. Sealing of the stems did not cause trouble of this kind.

An electrically heated oven, suitable for operation at temperatures up to $350^{\circ} \mathrm{C}$ and thermostatically controlled to 1 degree, was used to heat the apparatus.

For tests of short duration the sealed glass tubes were slid into pieces of pipe, capped at one end, which were lined up in the oven and were at oven temperature. The tubes were thus kept from breaking others of the same series in case of a failure, and the uncertain time lag involved in heating cold pieces of pipe to the temperature of the oven was eliminated.

The larger tubes, if they were to be heated much above $100^{\circ} \mathrm{C}$, were enclosed in steel pressure vessels and protected with gas under pressure, as will be described later.

When it was found that rhodium, ruthenium, and osmium were more readily dissolved by suitable acid mixtures than was iridium, this last-named metal was used in studying the effects on the rate of solution of variations in temperature, concentration of hydrochloric acid, and concentration of the oxidizing agent. ${ }^{2}$ It seemed entirely probable that conditions suitable for the solution of iridium would result in at least equally rapid attack of any alloy of platinum-group metals, whether a mineral or a manufactured product. Within the range of materials thus far tried, this expectation has been confirmed.

The iridium used for the study of rates of solution was in the form of sheet $0.075 \mathrm{~mm}$ thick. The sheet was cut into pieces about 3 by 5 $\mathrm{mm}$, which had a total surface of 30 to $40 \mathrm{~mm}^{2}$, neglecting the surface area of the edges. The amount of iridium dissolved was determined by weighing the pieces of iridium before and after exposure to the reaction mixtures. The volume of metal dissolved was calculated from the density and the loss in weight. This, in turn, divided by the surface area, yielded the average rate at which the surface was dissolved away during the period of exposure. Rates of solution are expressed in microns per hour. If all surfaces of each particle of metal are attacked simultaneously, the time required for the complete solution of a sample will depend on the thickness (effective diameter) of the largest particle in it, provided that all the particles in the sample are of equally refractory composition.

The periods of exposure of iridium to the reaction mixtures varied from $1 / 2$ hour to 24 hours. Within this range they were made as long as possible without risking perforation of the iridium sheet, which would have altered the total area too much. This variation in the period of exposure helped to keep the total amount of reaction more nearly constant and thus to minimize the effect of changes in composition of the reaction mixture. The shorter periods were subject to some uncertainty because the time required to bring the reaction vessel to the desired temperature was not precisely known. Tests on tubes containing small amounts of various substances of known melting points showed that tubes slid into a pipe preheated in the oven reached a temperature within $1^{\circ} \mathrm{C}$ of the oven temperature in 10 minutes.

${ }^{2}$ Although the pressures existing during the experimental work varied greatly, they are discounted from further discussion with the notation that the concentration of the components and the temperature of the system determine the pressure attained, since the volume is fixed. The pressure coefficients of reaction rates are usually very small, hence pressure variations as such were assumed not to affect the rates of attack significantly. 


\section{Journal of Research of the National Bureau of Standards}

The 4-mm tubes used for the observations on rates of attack had a capacity of about $2 \mathrm{ml}$. In most of the experiments $1 \mathrm{ml}$ of reaction mixture was used, but in some of those dealing with variations in the concentration of hydrochloric acid the volume was smaller. With these exceptions, which will be noted, variations in results from failure to keep constant the starting ratio of the volumes of the liquid and gas phases are believed to be smaller than those arising from uncontrolled variations in the composition of the reaction systems. In order to determine the change in the volumes of the liquid and gas phases as the temperature rose, one tube, half-filled with a mixture of 1 volume of fuming nitric acid (91 weight-percent of $\mathrm{HNO}_{3}$ ) and 20 volumes of hydrochloric acid (36 weight-percent of $\mathrm{HCl}$ ), was suspended within a heater made by winding resistance wire on a larger glass tube. The maximum increase in volume of the liquid was about 4 percent, at about $300^{\circ} \mathrm{C}$. Above that temperature the liquid decreased in volume. At about $390^{\circ} \mathrm{C}$ the meniscus disappeared, and other phenomena associated with the critical temperature were observed.

In many instances a measured volume (usually $1 \mathrm{ml}$ ) of acid mixture was transferred to the reaction tube by means of a pipet. In some experiments in which the composition of the reaction mixture was more closely controlled, the components were added by weight. To do this with chlorine and with hydrochloric acid mixtures prepared from water and hydrogen chloride, it was necessary to chill the tubes to the temperature at which the gases would liquefy. In such instances the tubes to be charged were cooled by a slush of carbon dioxide in a mixture of equal volumes of chloroform and carbon tetrachloride. For the condensation of dry hydrogen chloride gas, liquid air was tried as a direct coolant but its temperature was too far below the freezing temperature of hydrogen chloride $\left(-111^{\circ} \mathrm{C}\right)$ and only a snow, which plugged the small tubes used, could be obtained. Dry ice in ether, through which air is bubbled, was found to have an equilibrium temperature of $-95^{\circ} \mathrm{C}$ and hence $10^{\circ} \mathrm{C}$ below the temperature of condensation of hydrogen chloride. The fire hazards involved in sealing tubes over this mixture may be avoided by transferring the tube, after it is charged, to liquid air.

With this method of charging the tubes, chlorine and hydrogen chloride could not be added in the exact amounts desired. Instead, several mixtures were made so that the ratios would be in the desired range. The tubes were charged and then sealed by heating the open end until the glass flowed together, after which slight warming of the tube below the seal caused the seal to blow out into the desired rounded shape. This procedure made it possible to determine by gain in weight the amount of the volatile component which had been added.

The reactions by which metallic iridium is changed to a soluble chloride may be represented by the single reaction,

$$
\mathrm{Ir}+4 \mathrm{Cl}+2 \mathrm{Cl}^{-} \rightarrow \mathrm{IrCl}_{6}^{=} \text {. }
$$

To be dissolved, iridium must both be oxidized and be converted into the chloroiridate ion. The chloroiridite ion, $\mathrm{IrCl}_{6} \overline{\overline{6}}$, is another possible soluble form but does not appear as long as chlorine or an equivalent oxidant is present in excess. Chlorine in the presence of hydrochloric acid is an effective oxidant for iridium at temperatures from $250^{\circ}$ to $300^{\circ} \mathrm{C}$; in fact, the results of the present work show it to be just as effective as mixtures of hydrochloric acid with nitric acid, or 
with perchloric acid, of equal stoichiometric oxidizing power. ${ }^{3}$ Consequently, the latter reagents will be regarded merely as sources of chlorine, in accordance with the reactions,

$$
\begin{gathered}
7 \mathrm{HCl}+\mathrm{HClO}_{4} \rightarrow 4 \mathrm{Cl}_{2}+4 \mathrm{H}_{2} \mathrm{O} \\
10 \mathrm{HCl}+2 \mathrm{HNO}_{3} \rightarrow 5 \mathrm{Cl}_{2}+\mathrm{N}_{2}+6 \mathrm{H}_{2} \mathrm{O}
\end{gathered}
$$

The reaction between hydrochloric acid and perchloric acid is far from instantaneous, even at $300^{\circ}$, but the indicated products are the only ones that can be reasonably expected. The expression given for the reaction between hydrochloric acid and nitric acid is justified by an experiment in which an excess of finely divided iridium was heated at $300^{\circ}$ for 120 hours with a mixture of 20 volumes of hydrochloric acid and 1 volume of fuming nitric acid. In the resulting solution, no evidence was found of oxides of nitrogen, or of the ammonium radical or hydroxylamine. The pressure within the tube when opened at room temperature suggested the presence of nitrogen. Stoichiometric calculations indicated the stage of valence of the nitrogen to be not higher than zero. In this instance, with the oxidant entirely consumed, the dissolved iridium was partly in the form of $\mathrm{IrCl}_{6}^{\overline{\bar{*}}}$ and partly in the form of $\mathrm{IrCl}_{6}=$ ions.

Sodium chlorate was also used as an oxidant largely because it could be added conveniently as weighed amounts of the crystals. Its reaction with hydrochloric acid to form chlorine is very rapid, even at room temperature. Consequently, the acid in the tube had to be frozen before the chlorate was introduced.

The small variations in the ratio of the volume of liquid to that of vapor in the tubes, with consequent changes in the composition of the two phases, have already been mentioned. When temperature was the variable under consideration, the composition of both phases also varied considerably because of the changing partial vapor pressures of the components and because of the changing density of the liquid. The composition of the system varied further as the reaction proceeded, and to different extents if the total amount of reaction was not constant. At the optimum concentrations of chlorine a large part of the total amount of chlorine present was consumed in the reaction. On the other hand, the concentration of hydrogen chloride, which was always large relative to the amount of chloride ions consumed, was little affected.

When sodium chlorate was used as the oxidant, sodium chloride was present as an additional component. It should also be pointed out that the tubes contained about $1 \mathrm{ml}$ of air at atmospheric pressure. To the oxygen from this source must have been added some produced by the reaction between chlorine and water at the elevated temperatures. Finally, the formation of chloroiridate ions during the reaction introduced still another component into the system. For all these reasons the systems were much too complicated to permit considering the observed rates of attack as in any sense characteristic of completely defined systems. Instead, they were considered only as having relative significance and as pointing the way to combinations of temperature and composition of the acid mixtures that could be used to get optimum rates of solution for the practical purposes of chemical operations.

\footnotetext{
${ }^{3}$ In the case of platinum, molecular chlorine in the presence of hydrochloric acid does not appreciably attack the metal at temperatures up to $100^{\circ} \mathrm{C}$., whereas the familiar aqua regia does so readily, as do also mixtures of hydrochloric acid with certain oxidants other than nitric acid, even ferric chloride.
} 


\section{EXPERIMENTAL RESULTS}

\section{EFFECT OF VARIATION IN TEMPERATURE}

Preliminary experiments showed that mixtures containing a much smaller proportion of nitric acid than that commonly used in aqua regia ( 3 or 4 volumes of hydrochloric acid to 1 of nitric acid) were much more effective than aqua regia in dissolving iridium at elevated temperatures. A mixture of 20 volumes of concentrated hydrochloric acid (about 36 weight-percent of $\mathrm{HCl}$ ) with 1 volume of fuming nitric acid (about 91 weight-percent of $\mathrm{HNO}_{3}$ ) was used in many of the experiments in which nitric acid was the oxidant. With this mixture, the rate of solution of iridium was 0.05 micron per hour at $225^{\circ} \mathrm{C}$. Above this the rate of attack increased rapidly with rising temperature. At $255^{\circ} \mathrm{C}$ the observed rate was 0.44 ; at $275^{\circ} \mathrm{C}, 1.3$; and at $300^{\circ} \mathrm{C}, 2.2$ microns per hour. Above $300^{\circ} \mathrm{C}$ there was some conflict in the evidence about a continuing increase in rate of attack. Since $300^{\circ} \mathrm{C}$ was the maximum temperature that was convenient for operation with the facilities at hand, and since it was found to provide an adequate rate of attack by suitably chosen reaction mixtures, no detailed study was made of reaction rates at higher temperatures. From the figures given for the interval from $225^{\circ}$ to $300^{\circ} \mathrm{C}$ it will be seen that the variation of rate with temperature is similar to that observed for chemical reactions in general. Supporting evidence was obtained in two series of experiments, one at $250^{\circ}$ and one at $300^{\circ} \mathrm{C}$, in which the variable under study in each series was the concentration of chlorine. For comparable concentrations of chlorine the rate at $300^{\circ} \mathrm{C}$ was four or more times that at $250^{\circ} \mathrm{C}$.

\section{EFFECT OF VARIATION IN THE CONCENTRATION OF HYDROGEN CHLORIDE}

In a preliminary series of observations at $260^{\circ} \mathrm{C}$ the ratio of 1 volume of fuming nitric acid to 20 volumes of hydrochloric acid was maintained, but the strength of the hydrochloric acid was varied from 27 to 45 weight-percent of $\mathrm{HCl}$. This was done by mixing ordinary concentrated hydrochloric acid, which contains 35 to 36 weight-percent of $\mathrm{HCl}$, with water or with varying amounts of a fortified acid (45 weight-percent of $\mathrm{HCl}$ ), which was prepared by fractionally distilling: the ordinary acid and collecting the first fractions in a chilled receiver. The rates of attack observed with mixtures containing 27 and 30 weight-percent of $\mathrm{HCl}$ were one-fifth and one-half, respectively, of that observed with acid containing 35 weight-percent of $\mathrm{HCl}$. With mixtures containing 40 and 45 weight-percent of $\mathrm{HCl}$ the rates were, respectively, 5 and 8 times that of the solution containing 35 weightpercent of $\mathrm{HCl}$.

The total range in relative rates for these variations in strength of added hydrogen chloride was thus 1 to 40 . If all of the nitric acid added is assumed to react with hydrochloric acid to yield water, chlorine, and nitrogen, there is a drastic change in the effective concentration of hydrochloric acid in the mixture. On this assumption the concentration of hydrogen chloride changes from 27 to 11 , from 35 to 21 , and from 45 to 34 weight-percent of $\mathrm{HCl}$. On this basis the extreme range in final concentration of the acid, as weight-percent of $\mathrm{HCl}$, is from 11 to 34 , which corresponds to a threefold difference, instead of from 27 to 45 , in which the strongest acid is less than 
twice as concentrated as the weakest. When this work was done, the rates were not calculated in microns per hour, hence the results are comparable only among themselves and not with those observed in other series of experiments.

Several other series of observations were made in which $0.050 \mathrm{~g}$ of sodium chlorate was used as the oxidizing agent instead of $0.05 \mathrm{ml}$ of fuming nitric acid. This amount of sodium chlorate yields $0.100 \mathrm{~g}$ of

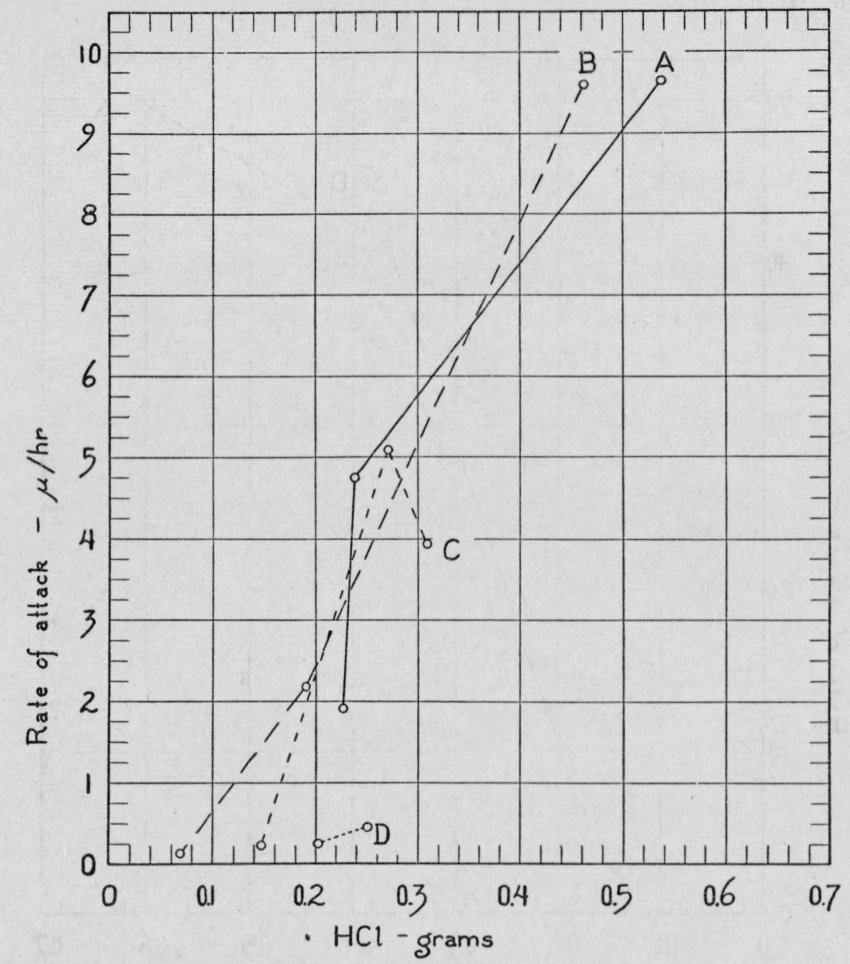

FIGURE 2.-Effect of the amount of hydrogen chloride present after reaction with $0.05 \mathrm{~g}$ of $\mathrm{NaClO}_{3}$ on the rate of solution of iridium at approximately $250^{\circ} \mathrm{C}$.

[On page 369 is given a tabulation of the conditions under which the data were obtained]

chlorine by reaction with hydrochloric acid. The reaction mixtures were prepared in each tube by measuring or weighing-in definite amounts of water, chlorate, and hydrogen chloride. The results obtained at approximately $250^{\circ} \mathrm{C}$ are shown in figure 2 . In this figure the concentration is given" in terms of the effective hydrogen chloride, that is, the weight of added hydrogen chloride corrected for that used to produce chlorine by reaction with sodium chlorate. The following are the conditions under which these observations were made.

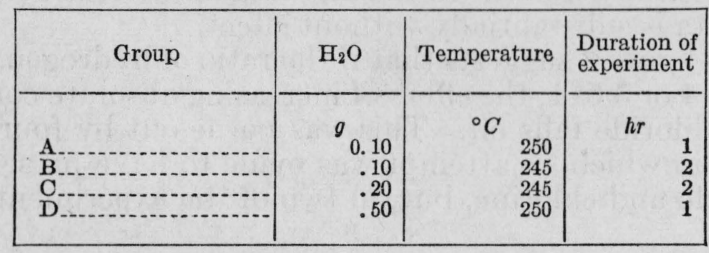


The conditions under which the results of groups $A$ and $B$ were obtained can be considered substantially identical. The relatively close agreement of the results of group $C$ with those in $A$ and $B$ indicate that the ratio of hydrogen chloride to water is much less important than the absolute concentration of hydrogen chloride. However, the two results in group $D$, obtained with a much different ratio of hydrogen chloride to water, show that the relative concentration has some bearing on the rate of attack.

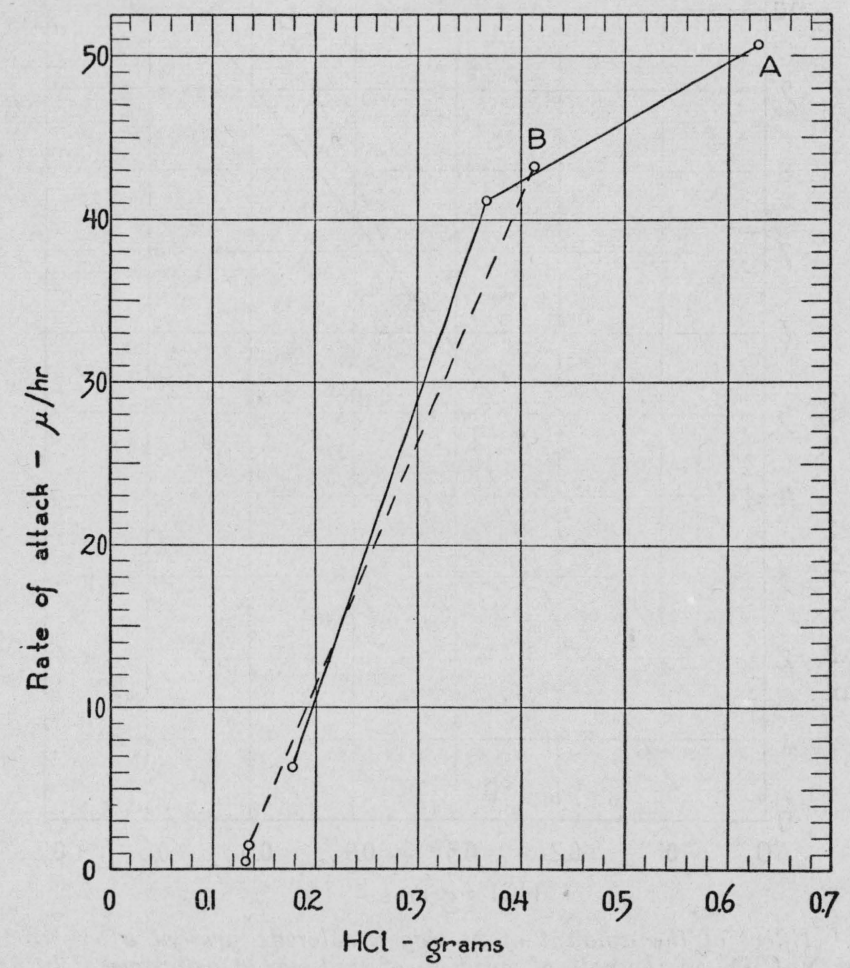

FiguRE 3.-Effect of the amount of hydrogen chloride after reaction with $0.05 \mathrm{~g}$ of $\mathrm{NaClO}_{3}$ on the rate of solution of iridium at $300^{\circ} \mathrm{C}$.

Total time $1 / 2 \mathrm{hr}$. Amount of $\mathrm{H}_{2} \mathrm{O}$ : Curve $A, 0.1 \mathrm{~g}$; curve $B, 0.2 \mathrm{~g}$.

Figure 3 shows results obtained at $300^{\circ} \mathrm{C}$. The concentration of acid is again expressed as the effective weight of hydrogen chloride in the system at the beginning of the period. Figure 3 shows that the rate of attack at $300^{\circ} \mathrm{C}$ increases rapidly with increasing hydrogen chloride content. Comparison with figure 2 shows that the change of rate is about 5 times as great at $300^{\circ}$ as at $250^{\circ} \mathrm{C}$, as described in a previous section. The variation in the ratio of hydrogen chloride to water in the two sets of data is substantially without effect.

The data in figure 3 suggests that if the ratio of hydrogen chloride to water exceeds 4 or 5 to 1 , the effect of increasing absolute concentration of hydrogen chloride falls off. This was borne out by four trials with iridium sheet in which an attempt was made to have present only hydrogen chloride and chlorine, but, in two of the experiments, traces of 
water were accidently introduced. These two showed slightly higher rates than the two in which water was thought to be absent, but the rates for the entire series were very low. They ranged from 0.008 to 0.3 micron per hour. It is quite evident that some water must be present for the reaction to proceed at an appreciable rate. It appears that the presence of moderate amounts of water does not appreciably affect the rate; but there are indications that too much water, that is, more water than hydrogen chloride, may reduce the rate.

In an attempt to learn whether the rate of attack observed with hydrogen chloride might reflect the effect of chloride ions rather than of hydrogen chloride, a series was tried in which lithium chloride was used to provide part or all of the chloride ions. In acid mixtures containing 15 and 21 weight-percent of $\mathrm{HCl}$ with enough lithium chloride added to make the chloride ion concentration equivalent to that of 36 weightpercent of $\mathrm{HCl}$, the results obtained were approximately the same as those with 36 weight-percent of $\mathrm{HCl}$. A solution containing the same chloride ion concentration provided entirely by lithium chloride showed no attack of the iridium whatever, but a similar one containing 1 to 2 weight-percent of $\mathrm{HCl}$ showed an appreciable rate of attack. The maximum amount of lithium chloride that could be substituted for hydrogen chloride without markedly affecting the rate of attack was not determined.

A few trials were also made of additions of ammonium chloride, potassium chloride, and sodium chloride, but only in moderately small amounts because of the limited solubility of these substances in the acid mixture. It was not possible to draw definite conclusions from these trials, but they suggest that further study of such systems might be of interest in developing procedures that would involve total pressures much lower than those encountered when hydrochloric acid is used alone.

In the study of the rate of attack as a function of concentration of hydrochloric acid the highest rate observed was 51 microns per hour, with a water-hydrogen chloride mixture containing 84 weight-percent of the latter, at $300^{\circ} \mathrm{C}$. At this rate a period of 20 hours would be long enough to dissolve a piece of iridium $2 \mathrm{~mm}$ thick. It is true that an acid mixture of that strength is hardly practicable in ordinary analytical work. On the other hand, the liquid-vapor ratio was considerably below the optimum. Likewise, the chlorine concentration ( $0.1 \mathrm{~g}$ of chlorine per $\mathrm{ml}$ of acid) was much higher than the optimum, as will be shown in the next section. By proper selection of conditions, rates of at least 25 microns per hour can be attained without the use of fortified hydrochloric acid.

\section{VARIATION IN THE CONCENTRATION OF CHLORINE}

It has already been noted that a mixture of one volume of fuming nitric acid (91 weight-percent of $\mathrm{HNO}_{3}$ ) with 20 volumes of hydrochloric acid (36 weight-percent of $\mathrm{HCl}$ ) was found to attack iridium much more rapidly than the usual aqua regia mixture. The mixture containing the smaller proportion of nitric acid dissolved iridium 20 times as fast as did ordinary aqua regia. When the ratio of nitric acid to hydrochloric acid was further decreased, to $1: 40$, the rate of attack again increased, by a factor of 3. A partial explanation lies in the fact that the reaction between the two acids at the high temper-

$615691-44-5$ 
atures of the experiments results in an increase in the amount of water in the system (in proportion to the amount of oxidant added) and a corresponding decrease in the amount of hydrogen chloride. Hence, the loss in concentration of the hydrochloric acid is less with the smaller proportion of oxidant. If the reaction yields water, chlorine, and nitrogen, the strength of the hydrochloric acid in the $1: 20$ and $1: 40$ mixtures mentioned above can be regarded as changing from an initial concentration of 36 weight-percent of $\mathrm{HCl}$ to 20 and 28 , respectively.

Later experiments, in which chlorine, as such, was used as the oxidant, thus maintaining a constant amount of hydrogen chloride in the system except for the small amount consumed by the formation of $\mathrm{IrCl}_{\overline{6}}^{-}$ions, showed that the rate of attack varied markedly as a function of the concentration of chlorine. ${ }^{4}$ Four groups of these

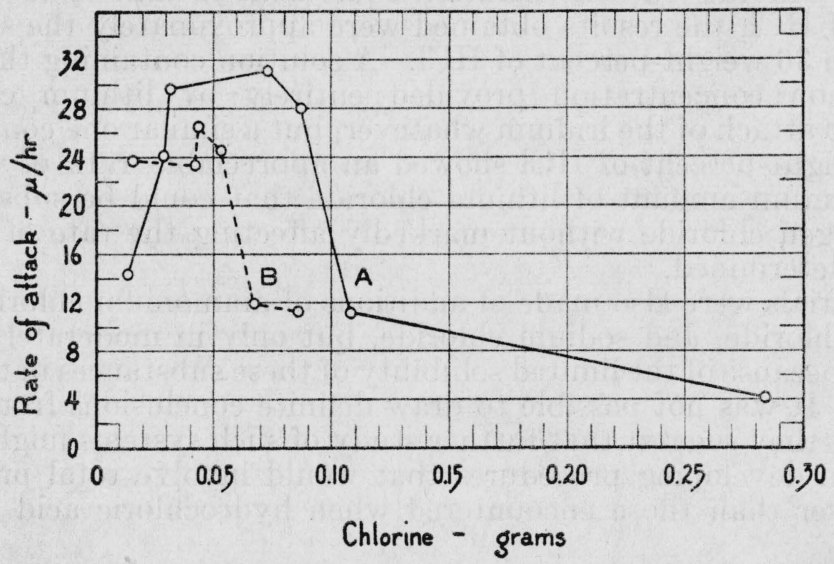

FIGURE 4.-Effect of the amount of chlorine on the rate of solution of iridium in $1 \mathrm{ml}$ of 36 weight-percent of $\mathrm{HCl}$ at $300^{\circ} \mathrm{C}$.

Curve $A$, for $1 \mathrm{hr}$; curve $B$, for $1 / 2 \mathrm{hr}$.

observations are shown in figures 4 and 5 . In the experiments recorded in figure 4, the conditions kept constant were the quantity of hydrochloric acid and the temperature. The three higher rates shown in series $A$ are lower than the true values for the corresponding concentrations of chlorine because the pieces of iridium were nearly destroyed, with a consequent marked decrease in surface area toward the end of the period of attack.

Figure 5 shows two sets of results obtained with $1 \mathrm{ml}$ of acid in a 2-ml tube, heated for one-half hour at $300^{\circ} \mathrm{C}$. Chlorine, $A$, and sodium chlorate, $B$, were used as the oxidants. The strength of the hydrochloric acid, after allowing for that consumed by the reactions with chlorate, was 28 weight-percent (corresponding to $0.35 \mathrm{~g}$ of $\mathrm{HCl}$ in the system). To correlate these results with the preliminary observations on varying amounts of nitric acid it should be pointed out that $1 \mathrm{ml}$ of a 1:40 mixture of hydrochloric-nitric acid would contain the equivalent of $0.1 \mathrm{~g}$ of chlorine. Thus, the results obtained previously with that mixture should correspond approximately to those obtained with the largest concentration of chlorine shown in figure 5. Although the

\footnotetext{
- Although the chlorine formed a separate liquid phase in these systems at room temperature the extra phase disappeared much below $300^{\circ} \mathrm{C}$.
} 
variations shown in figures 4 and 5 are not as regular as might be desired, it appears that, beginning with very low concentrations, there is at first a rapid increase in the rate of solution to a fairly well-defined maximum, after which the rate declines sharply at first and then more gradually. The same behavior is shown in figure 6 , in which are given the results of a group of observations similar to the others, except that the temperature was $250^{\circ} \mathrm{C}$. The period was 1 hour. The oxidant

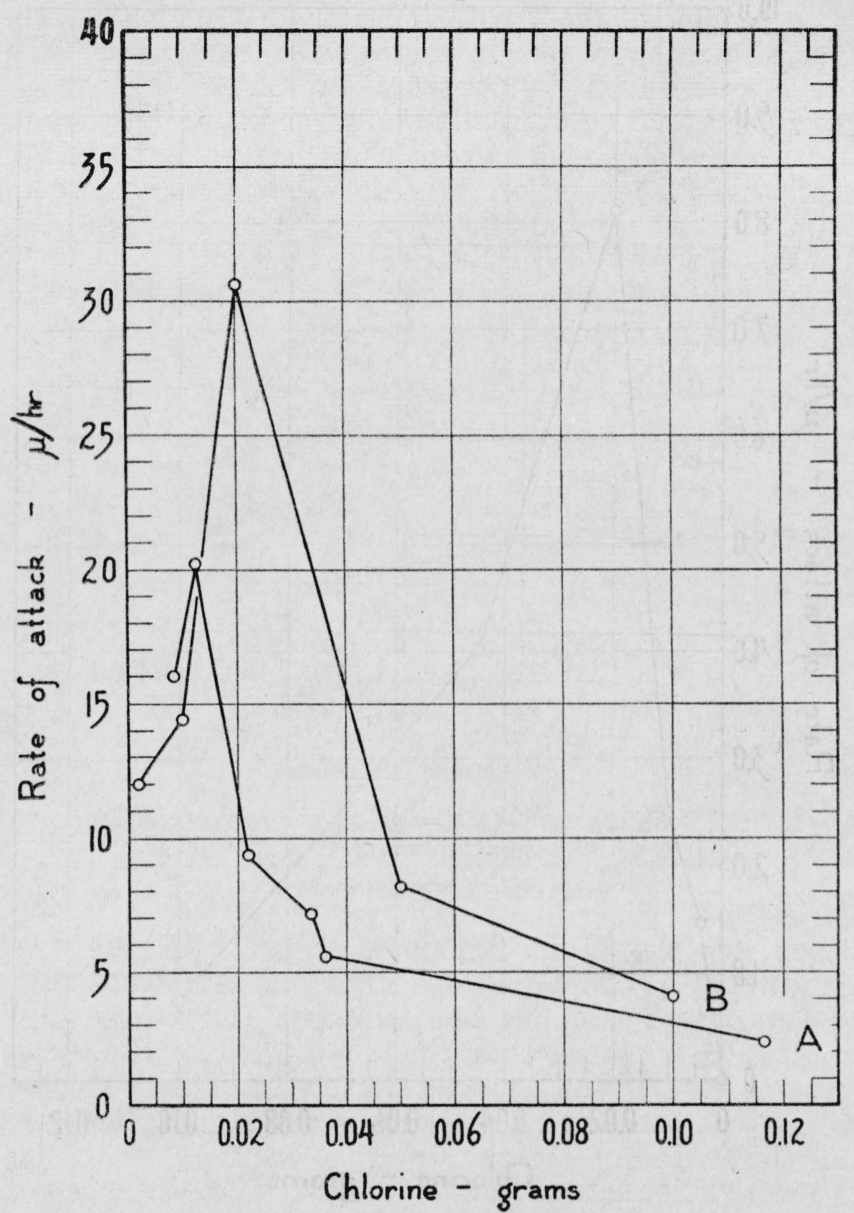

FIGURe 5.-Effect of the amount of chlorine on the rate of solution of iridium with constant concentration of $\mathrm{HCl}$ when heated $1 / 2$ hour at $300^{\circ} \mathrm{C}$.

Curves, $A, 1 \mathrm{ml}$ of 28 weight-percent $\mathrm{HCl}$ with added chlorine; $B$, solution equivalent to $A$, but with rhe chlorine supplied by additions of $\mathrm{NaClO}_{3}$.

was sodium chlorate. In this group the net hydrogen chloride content of the acid varied, because no adjustment was made for the acid used by reaction with chlorate, from 36 weight-percent for the lowest concentration of chlorine to 28 weight-percent for the highest. In this series sodium chloride was added in varying amounts so that the total sodium chloride content, taking into account that yielded by the sodium chlorate, was constant. This was done to obviate any possible effect of changes in the concentration of this component. 
A few experiments were performed with perchloric acid as the oxidant. In this system, and in the nitric acid-hydrochloric acid system, all the oxidant is not immediately available as chlorine. Consequently, the variation in rate of attack with varying concentration of oxidant is not directly comparable with the results obtained with chlorine and with sodium chlorate.

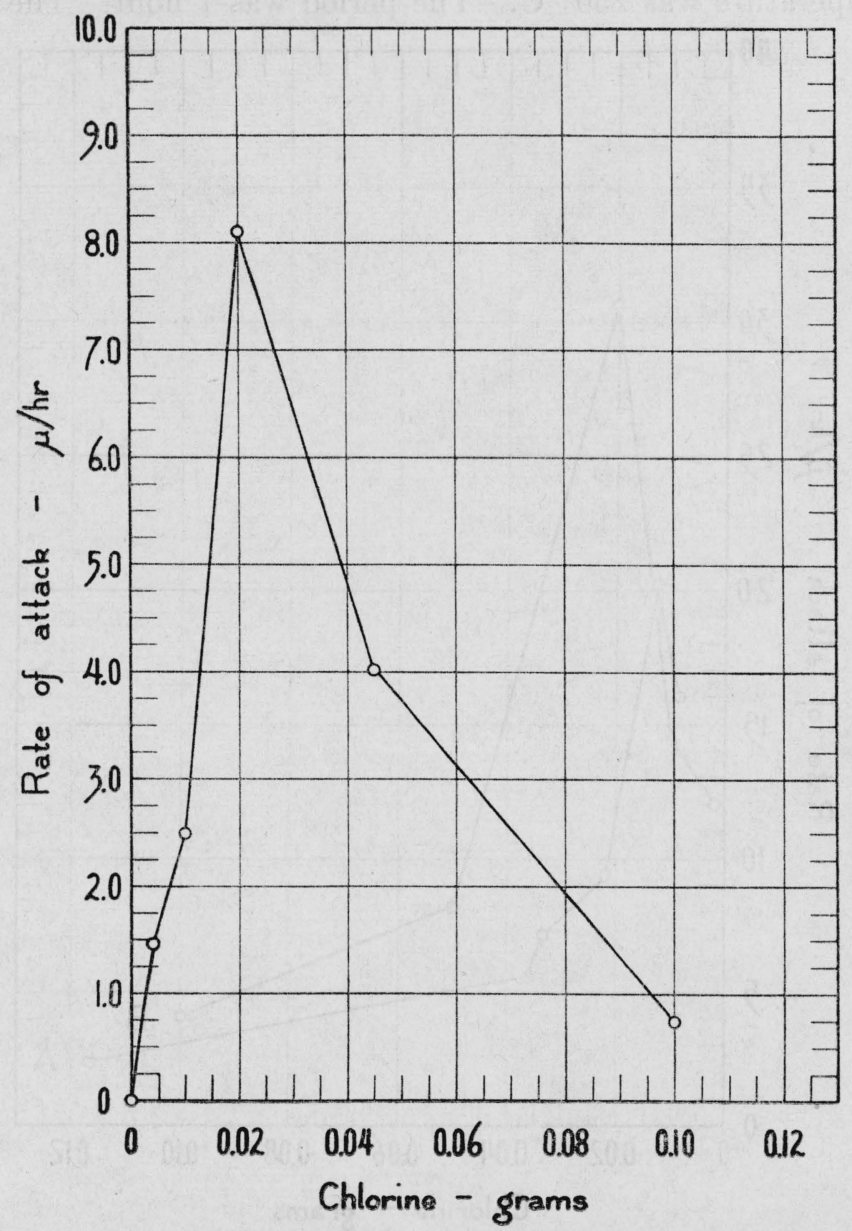

FIGURE 6.-Effect of the amount of chlorine, produced from sodium chlorate, on the rate of solution of iridium, in $1 \mathrm{ml}$ of 36 weight-percent $\mathrm{HCl}$, when heated 1 hour at $250^{\circ} \mathrm{C}$.

The amount of chlorine consumed by reaction with iridium at low concentrations of chlorine caused a change in its concentration, since the iridium surface was large compared with the amount of reaction mixture. In figure 6 , for example, in the experiment that gave the highest rate, the amount of chlorine left at the end of the reaction period was $0.017 \mathrm{~g}$, as against $0.020 \mathrm{~g}$ at the beginning of the period. The results of similar experiments in tubes of larger capacity, where the volume of reaction mixture was five times as great, but with the 
same area of iridium and the same concentration of chlorine in the reaction mixture, are shown by curves $B$ and $C$ in figure 7 .

Figures 4 to 7 , inclusive, show that careful attention must be given to the concentration of chlorine in determining the optimum conditions for the solution of iridium. These figures indicate that the optimum concentration is in the range 0.02 to $0.04 \mathrm{~g}$ of chlorine per

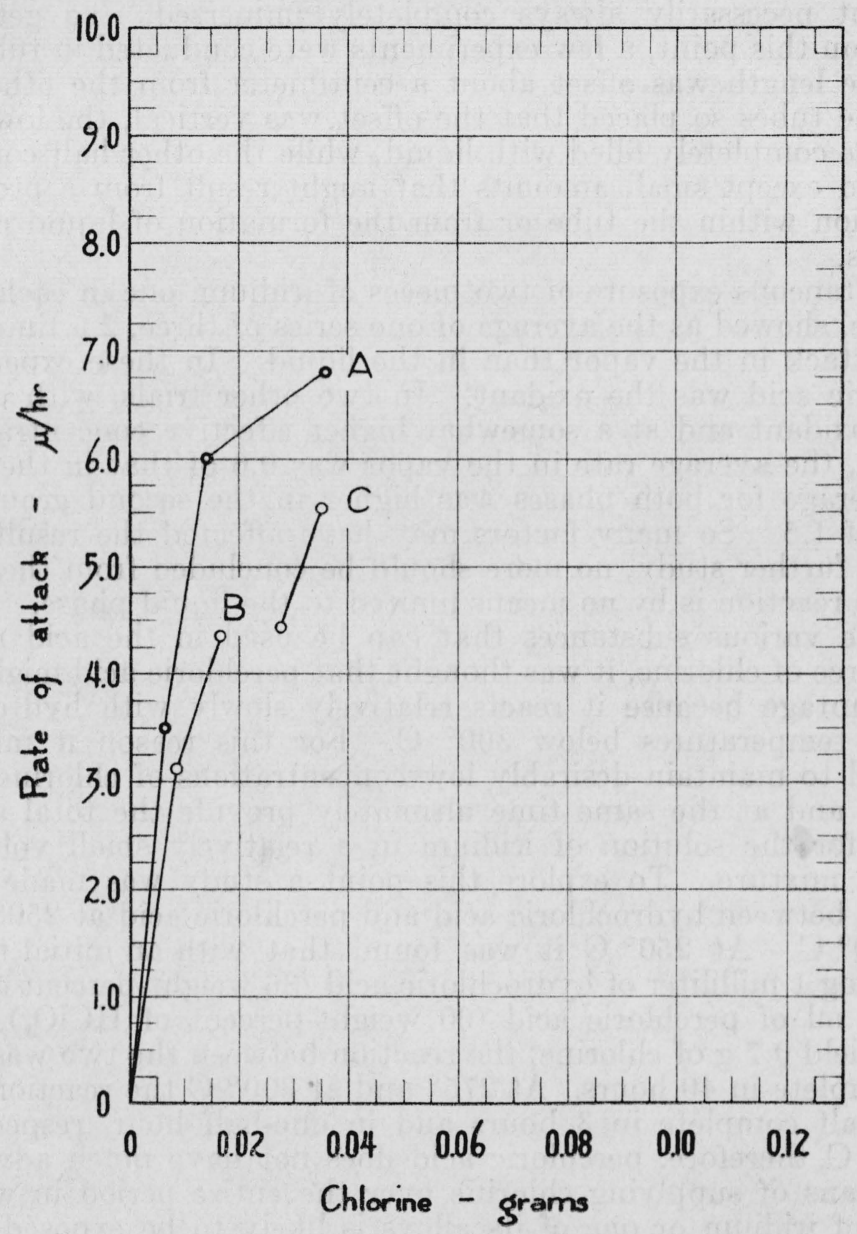

FIGURE 7.-Comparison of the initial rates of solution of iridium at the lower concentrations of oxidant with larger amounts of reaction mixture.

$A$, Volume of solution used was $1 \mathrm{ml}$ of 36 weight-percent $\mathrm{HCl}$ plus the chlorine. Heated 1 hour at $250^{\circ} \mathrm{C}$; $B$, volume of solution used was $5 \mathrm{ml} 36$ weight-percent of $\mathrm{HCl}$ plus the chlorine. Heated 1 hour at $251^{\circ} \mathrm{C}$; $C$, volume of solution used was $5 \mathrm{ml} 36$ weight-percent of $\mathrm{HCl}$ plus the chlorine. Heated 2 hours at $251^{\circ} \mathrm{C}$.' For comparison with other curves, the amounts of chlorine used in obtaining curves $B$ and $C$ have been divided by 5 and plotted as the grams of chlorine per $2 \mathrm{ml}$ capacity of reaction chamber.

milliliter of hydrochloric acid. If an initial concentration of 0.04 $\mathrm{g} / \mathrm{ml}$ is used, the amount of reaction mixture should be adjusted to the amount of iridium to be dissolved, so that the final concentration of chlorine will not be much less than $0.02 \mathrm{~g} / \mathrm{ml}$. 


\section{MISCELLANEOUS OBSERVATIONS}

In view of what has been said earlier about variation of composition in the liquid and gas phases, it may be of interest to note that the reaction should not be considered as occurring in the liquid phase only. The reaction tubes were placed in the oven horizontally, but since they were never more than half full of liquid the pieces of iridium were not necessarily always completely immersed. To get information on this point, a few experiments were conducted in tubes half of whose length was offset about a centimeter from the other half. With the tubes so placed that the offset was vertical, the lower half would be completely filled with liquid, while the other half contained no liquid except small amounts that might result from a process of distillation within the tube or from the formation of liquid reaction products.

Simultaneous exposure of two pieces of iridium, one in each end of the tube, showed as the average of one series of three, 2.5 times more rapid attack in the vapor than in the liquid. In these experiments perchloric acid was the oxidant. In two other trials, with chlorine as the oxidant and at a somewhat higher effective concentration of chlorine, the average rate in the vapor was 0.6 of that in the liquid. The average for both phases was higher in the second group by a factor of 1.5. So many factors may have affected the results that, without further study, no more should be concluded from them than that the reaction is by no means limited to the liquid phase.

Of the various substances that can be used in the acid mixture as a source of chlorine, it was thought that perchloric acid might have an advantage because it reacts relatively slowly with hydrochloric acid at temperatures below $300^{\circ} \mathrm{C}$. For this reason it might be expected to maintain desirably low concentrations of chlorine in the mixture and at the same time ultimately provide the total amount needed for the solution of iridium in a relatively small volume of reaction mixture. To explore this point a study was made of the reaction between hydrochloric acid and perchloric acid at $250^{\circ}, 275^{\circ}$, and $300^{\circ} \mathrm{C}$. At $250^{\circ} \mathrm{C}$ it was found that with an initial mixture containing 1 milliliter of hydrochloric acid (36 weight-percent of $\mathrm{HCl}$ ) and $0.1 \mathrm{ml}$ of perchloric acid (60 weight-percent of $\mathrm{HClO}_{4}$ ), which would yield $0.7 \mathrm{~g}$ of chlorine, the reaction between the two was about half complete in 40 hours. At $275^{\circ}$ and at $300^{\circ} \mathrm{C}$ the reactions were about half complete in 3 hours and in one-half hour, respectively. At $300^{\circ} \mathrm{C}$, therefore, perchloric acid does not have much advantage as a means of supplying chlorine over the entire period in which a sample of iridium or one of its alloys is likely to be exposed to the reaction mixture. At lower temperatures it may be expected to act in the desired manner, unless its reaction with hydrochloric acid is catalyzed by the iridium or the reaction products. It has the very real advantage of not reacting at all with hydrochloric acid at room temperature. Thus it can be added to hydrochloric acid without making it necessary to chill the tube before sealing, as must be done if sodium chlorate or chlorine is added. Tubes containing nitric acid and hydrochloric acid in the proportion suitable for this work can be sealed without chilling. However, nitric acid may possibly have a specific disadvantage because of its tendency to introduce nitroso 
groups into the chloro complexes of certain platinum metals, notably ruthenium.

A few trials of hydrogen peroxide as the oxidant suggested that it also acted as a source of chlorine by reaction with hydrochloric acid, but the rate of attack on iridium was less than was obtained with other sources of chlorine. It is possible that some of the peroxide dissociates to form water and oxygen and that the oxygen does not in turn come to equilibrium with hydrogen chloride to form water and chlorine during the period of the experiment. In one instance this was 16 hours at $250^{\circ} \mathrm{C}$ and in another 1 hour at $300^{\circ} \mathrm{C}$. Hydrogen peroxide has further disadvantages in that, for a given yield of chlorine, its reaction with hydrochloric acid produces twice as much water as does perchloric acid, and that the strongest commercially available reagent contains 70 percent of water, whereas perchloric acid contains only about half as much and sodium chlorate none.

Bromine, in hydrochloric acid solution, was found to attack iridium considerably less readily than chlorine. An even smaller rate of attack by iodine was not distinguished with certainty from that produced by the air present. So far as they went, the experiments with bromine seemed to indicate a nearly constant rate of attack over a range of concentrations equivalent to 0.05 to $0.20 \mathrm{~g}$ of chlorine, the range in which the rate of attack by chlorine rapidly declines with increasing concentration.

Ferric chloride, in a single experiment, produced a significant rate of attack. Experiments by McCaughey ${ }^{5}$ show that the rate of attack on gold by solutions of ferric and cupric salts increased with increasing concentrations of the oxidants (in a low range of concentrations). This might be of interest, in view of the relatively low oxidation potential of ferric chloride, in studying the mechanism of the reaction by which iridium is dissolved, but it was not further explored.

Oxygen (other than that in the air remaining in the tubes after sealing) was not used in this investigation as the oxidant. However, Tronev and Bondin ${ }^{67}$ have made some studies on the solution of platinum, iridium, and rhodium under higher air pressures. In their investigation the increase of pressure is really an increase in concentration of the oxidant. The maximum amount they used was stoichiometrically equivalent to about $0.04 \mathrm{~g}$ of chlorine in a $2-\mathrm{ml}$ tube.

Bromine in hydrobromic acid solution (48 weight-percent of $\mathrm{HBr}$ ) was also tried, but the rate of attack was considerably less than that with equivalent concentrations of chlorine and hydrochloric acid. In one trial, a small quantity of potassium iodide was added to a system containing hydrochloric acid and chlorine, but no significant effect was produced. In this system the end product is a compound of trivalent iridium rather than of the quadrivalent metal. There is no apparent advantage gained by substituting bromo- or iodo-complexes for the chloroiridate ion as the end product of the reaction.

\section{PREPARATION OF SAMPLES FOR ANALYSIS}

Among the materials on hand suitable for practical trials of the new procedure was a granular, impure iridium containing particles up to

6 W. J. McCaughey, J. Am. Chem. Soc. 31, 1261-70 (1909).

- V. G. Tronev, and S. M. Bondin, Ann, secteur platine, Inst. chim. gên. (USSR) 15, 113-23 (1938).

7 V. G. Tronev, Compt. rend, acad. sci. URSS 15, 555-8 (1937) in German. A resumé of the preceding reference. 
nearly a millimeter thick. This material has the interesting history of having been recovered over a considerable period of time from the anode residues obtained during the electrolytic refining of gold in the United States Assay Office at New York. It contains some ruthenium but virtually no other platinum metals. To get it into solution for analysis would ordinarily pose a difficult problem. During the early trials of the procedure $1.5 \mathrm{~g}$ of this material was attacked with 15 $\mathrm{ml}$ of a fortified hydrochloric acid (47-48 weight-percent of $\mathrm{HCl})$ and $0.75 \mathrm{ml}$ of fuming nitric acid (91 weight-percent of $\mathrm{HNO}_{3}$ ). This was done in a 15-mm Pyrex tube protected by a compensating pressure of 3,500 to $4,000 \mathrm{lb} /$ in. $^{2}$ in a steel shell. After heating for 24 hours at $300^{\circ}$ $\mathrm{C}$ a few undissolved grains were still visible. After the tube was heated 2 days longer at $300^{\circ} \mathrm{C}$ there was left one tiny grain of metal and a few grains of sandy, nonmetallic material. Although the fortified hydrochloric acid in this early trial was favorable to a rapid rate of attack, the quantity of nitric acid used was very much larger than the optimum.

A 2-g sample of the same material was heated at $300^{\circ} \mathrm{C}$ with $20 \mathrm{ml}$ of hydrochloric acid ( 36 weight-percent of $\mathrm{HCl}$ ) and about $2 \mathrm{~g}$ of chlorine. After 24 hours there was a small residue. In this instance also the amount of oxidant used was too large.

Samples of about $1.5 \mathrm{~g}$ of a coarse-grained California mineral, which subsequent analysis showed to contain approximately equal amounts of iridium, osmium, ruthenium, and platinum (with minor amounts of rhodium and iron), were treated with $20 \mathrm{ml}$ of hydrochloric acid (36 weight-percent of $\mathrm{HCl}$ ) and $1 \mathrm{~g}$ of sodium chlorate (equivalent to $2 \mathrm{~g}$ of chlorine) at $300^{\circ} \mathrm{C}$ for 24 hours. Solution was complete. Nuggets of an Alaskan mineral high in iridium, weighing from 1.1 to $1.6 \mathrm{~g}$, also were completely dissolved in a mixture of $15 \mathrm{ml}$ of hydrochloric acid (36 weight-percent of $\mathrm{HCl}$ ) and 0.4 to $0.7 \mathrm{ml}$ of a solution of 60 weightpercent of perchloric acid when heated at $300^{\circ} \mathrm{C}$ for 24 hours.

In this laboratory the mixture commonly used to dissolve ordinary platinum in open vessels consists of 4 volumes of hydrochloric acid (36 weight-percent of $\mathrm{HCl}$ ), 1 volume of water, and 1 volume of nitric acid (70 weight-percent of $\left.\mathrm{HNO}_{3}\right)^{8}{ }^{8}$ This is not the most favorable mixture for use in sealed vessels at about $100^{\circ} \mathrm{C}$ as will be seen from table 1. A companion experiment, in which a mixture of $1 \mathrm{ml}$ of

TABLE 1.-Rates of attack of pure platinum at $100^{\circ} \mathrm{C}$ with different mixtures of hydrochloric and nitric acid

\begin{tabular}{|c|c|c|c|}
\hline \multicolumn{3}{|c|}{$\begin{array}{c}\text { Acid mixture, parts by } \\
\text { volume }\end{array}$} & \multirow{2}{*}{$\begin{array}{l}\text { Rate of } \\
\text { attack }\end{array}$} \\
\hline $\mathrm{HCl}$ & $\mathrm{HNO}_{3}$ : & $\mathrm{H}_{2} \mathrm{O}$ & \\
\hline $\begin{array}{r}4 \\
4 \\
8 \\
20 \\
100\end{array}$ & $\begin{array}{l}1 \\
1 \\
1 \\
1 \\
1\end{array}$ & \begin{tabular}{c}
1 \\
1 \\
\\
\hdashline... \\
\end{tabular} & $\begin{array}{c}\text { Microns/hr } \\
100 \\
157 \\
167 \\
119 \\
26\end{array}$ \\
\hline
\end{tabular}

- The nitric acid used in the first mixture was the ordinary acid (70 weight-percent of $\mathrm{HNO}_{3}$ ). In the others, fuming acid (91 weight-percent of $\mathrm{HNO}_{3}$ ) was used.

\footnotetext{
8 This mixture, which involves slight dilution with water, has very nearly the hydrogen chloride concentration of constant-boiling hydrochloric acid and hence is more economical to use on a large scale than the undiluted mixture.
} 
hydrochloric acid and $0.05 \mathrm{~g}$ of sodium chlorate was used, showed a rate of attack of 167 microns per hour.

Platinum alloyed with as little as 10 percent of iridium is dissolved only slowly by aqua regia in open vessels at the temperature of the steam bath. In the course of analyzing a large number of samples of platinum-iridium alloys it has been found very useful to seal the samples (weighing about $0.5 \mathrm{~g}$ and in the form of chips 0.1 to $0.2 \mathrm{~mm}$ thick) in $8-\mathrm{mm}$ tubes with $5 \mathrm{ml}$ of a mixture of 8 to 10 volumes of hydrochloric acid and 1 volume of nitric acid. When the tubes are heated overnight at about $110^{\circ} \mathrm{C}$, solution is accomplished with a minimum of inconvenience. At $110^{\circ} \mathrm{C}$ tubes of this size, of standard wall thickness, need no external protection.

\section{BEST OPERATING CONDITIONS}

On the basis of the foregoing discussion and of other experience in this laboratory, recommendations can be made as to desirable conditions for the solution of refractory platiniferous materials in hydrochloric acid mixtures at elevated temperatures. By a suitable choice of conditions, samples to be analyzed can be converted into a solution of chloro-compounds in a small volume of acid, without contamination, without loss of volatile reaction products such as osmium tetroxide, and without the use of unusual equipment other than the steel shell in which the larger tubes must be placed to protect them against excessive internal pressures.

\section{QUANTITY AND STRENGTH OF HYDROCHLORIC ACID}

For most materials ordinary concentrated hydrochloric acid (about 36 weight-percent of $\mathrm{HCl}$ ) is satisfactory. For unusually large particles or for exceptionally refractory materials it is desirable to use a stronger acid. Concentrations up to about 50 weight-percent of $\mathrm{HCl}$ are conveniently prepared by distilling some of the ordinary acid through a condenser cooled with very cold water into a receiver packed in ice. Ratios of hydrogen chloride to water, higher than 1 to 1 , can be prepared by condensing dry hydrogen chloride on top of frozen water. The amount of acid used must be sufficient to produce the best ratio of acid to oxidant which is $20 \mathrm{~g}$ of $\mathrm{HCl}$ to $1 \mathrm{~g}$ of $\mathrm{Cl}_{2}$.

Not less than $4.2 \mathrm{~g}$ of $\mathrm{HCl}$ should be used for each gram of sample. If ordinary acid is used, $10 \mathrm{ml}$ of acid per $\mathrm{g}$ of sample is the minimum. A larger quantity, up to $20 \mathrm{ml}$ per $\mathrm{g}$, will hasten complete solution.

\section{NATURE AND QUANTITY OF OXIDIZING AGENT}

The best quantity of oxidizing agent for the solution of iridium at $250^{\circ}$ to $300^{\circ} \mathrm{C}$ is $0.025 \mathrm{~g}$ of $\mathrm{Cl}_{2}$ per milliliter of internal tube volume, provided as chlorine or by the action of any chlorine-producing oxidant.

Perchloric acid, ${ }^{9}$ preferably of 70 weight-percent, is the most convenient oxidant to use if the reaction is to be conducted at temperatures above $250^{\circ} \mathrm{C}$. At lower temperatures it reacts too slowly with hydrochloric acid to be suitable, and nitric acid or sodium chlorate should be used instead, the latter only if there is some reason to avoid the

\footnotetext{
$\overline{0 \text { WARNING.-If }}$ organic matter is present there is danger of explosion by reaction with the perchloric acid.
} 
possible formation of nitroso compounds, such as $\left(\mathrm{RuNOCl}_{5}\right)=$. For each gram of sample consisting predominantly of iridium, osmium, or platinum, $0.22 \mathrm{ml}$ of perchloric acid of 70 weight-percent of $\mathrm{HClO}_{4}$ should be used. If nitric acid is used as the oxidant, $0.27 \mathrm{ml}$ of the ordinary acid (70 weight-percent of $\mathrm{HNO}_{3}$ ) or $0.19 \mathrm{ml}$ of the fuming acid (91 weight-percent of $\mathrm{HNO}_{3}$ ) corresponds to the $0.22 \mathrm{ml}$ of perchloric acid solution. The corresponding weight of sodium chlorate is $0.37 \mathrm{~g}$. If the sample contains significant amounts of rhodium or ruthenium, a larger amount of oxidant should be used, in proportion to the smaller equivalent weight of these metals. It should be noted that if perchloric acid is used at temperatures much below $300^{\circ}$ some of it is likely to remain if the period of heating is not over 24 hours. Its presence may be undesirable in later analytical operations; for example, osmium or ruthenium tetroxide may be evolved if the solution is evaporated by heating.

For iridio-platinum alloys, which can be dissolved at temperatures as low as $100^{\circ}$ to $150^{\circ} \mathrm{C}$, perchloric acid is not recommended. For these alloys about $0.27 \mathrm{ml}$ of nitric acid (70 weight-percent of $\mathrm{HNO}_{3}$ ) per $\mathrm{g}$ of metal is desirable, or sodium chlorate, $0.37 \mathrm{~g}$ per $\mathrm{g}$ of metal, can be substituted.

\section{TEMPERATURE}

In general, the temperature at which the reaction is conducted should be no higher than is necessary to get complete solution in a convenient length of time, for example, in 18 to 24 hours. Temperatures as high as $300^{\circ} \mathrm{C}$ will be needed for only the most refractory materials, unless the sample particles are unusually large. Inspection of the tubes, after a trial period, will indicate whether the temperature need be raised to get complete solution within the desired time. As noted previously, perchloric acid probably is not satisfactory as the oxidizing agent if the reaction is to be conducted below $250^{\circ} \mathrm{C}$, or if there is organic matter present in the sample.

\section{DIMENSIONS OF REACTION TUBE}

The tube should be no larger than is necessary to accommodate, when half to two-thirds full, the quantity of acid to be used. For the amounts of acid needed for 1 to $1.5 \mathrm{~g}$ samples, heavy-walled Pyrex tubes of $15 \mathrm{~mm}$ bore, about $20 \mathrm{~cm}$ long, with 4- by 8-mm stems (see fig. 1) are very satisfactory. If larger samples are to be treated it will be desirable to increase the length of the tubes, rather than their diameter, or to divide the sample among several tubes.

\section{PROTECTION AND HANDLING OF THE SEALED TUBES}

The technique of filling, sealing, protecting and opening such tubes is described elsewhere. ${ }^{10}$ The filled and sealed tubes are placed in a steel shell, provided with a cap and gasket, into which previously has been placed sufficient calcium carbonate to neutralize all the acid in the tube in the unexpected event of its breaking. Solid carbon dioxide is then added in an amount calculated to give the desired pressure at the operating temperature. These approximate pressures to be expected

${ }_{10}$ C. L. Gordon, W. G. Schlecht, and E. Wichers, Use of sealed tubes for the preparation of acid solu. tions of samples for analysis or for small scale refining: Pressures of acids heated above $100^{\circ} C, \mathrm{~J}$. Research NBS 33, (1944) RP1622. 
by heating acid mixtures can be predicted as being nearly that of the hydrochloric acid used, provided the ratio of hydrochloric acid to oxidant is at least 10:1. Hence the use of hydrochloric acid of 23,37 , and 48 weight-percent $\mathrm{HCl}$ in the amount of slightly less than one-half $\mathrm{ml}$ in $1 \mathrm{ml}$ of internal capacity of the sealed tube may be expected at $300^{\circ} \mathrm{C}$ to reach pressures of at least $2,000,3,630$, and $4,730 \mathrm{lb} / \mathrm{in} .{ }^{2}$, respectively. The amounts of solid carbon dioxide that produce these pressures at $300^{\circ} \mathrm{C}$ are $0.14,0.24$, and, $0.31 \mathrm{~g}$ of $\mathrm{CO}_{2}$ per $\mathrm{ml}$ of free space surrounding the sealed tube.

After the carbon dioxide has been introduced the cap is quickly screwed on and the shell is immersed in water to test for leakage of the carbon dioxide around the gasket. The shell is then tilted gently a few times to distribute the sample within the tube, then placed horizontally in an oven and heated to the desired temperature. After cooling and removing the tube from the shell, it is chilled by immersion in a slush of dry ice in a 1:1 mixture of chloroform with carbon tetrachloride. This lowers the internal pressure sufficiently so that the tube can be opened without danger.

If osmium or ruthenium is thought to be present, immerse the opened end of the tube in a solution of approximately 20 weight-percent of $\mathrm{HCl}$ saturated with sulfur dioxide, and allow the contents of the tube to drain into this solution, so that the volatile oxides of ruthenium and osmium will not escape. If this is done, the solution should be evaporated and digested with hydrochloric acid to decompose sulfite complexes of the platinum metals before proceeding with the analysis.

Washington, May 25, 1944. 